\title{
ESTIMACIÓN DEL ÁREA FOLIAR DEL ARÁNDANO (Vaccinium corymbosum) POR MEDIO DE UN MÉTODO NO DESTRUCTIVO
}

\section{A NON DESTRUCTIVE METHOD FOR ESTIMATING THE LEAF AREA OF BLUEBERRY (Vaccinium corymbosum)}

\author{
Marco Cabezas-Gutiérrez ${ }^{1}$, Fernando Peña-Baracaldo ${ }^{2}$ \\ ${ }^{1}$ I.A., M.Sc., c. Ph.D. Docente investigador Facultad Ing. Agronómica U.D.C.A (hasta julio de 2011); ${ }^{2}$ I.A., M.Sc. Docente \\ investigador Facultad Ing. Agronómica U.D.C.A, Calle 222 No. 55-37. Bogotá, D.C. fepena@udca.edu.co
}

Rev. U.D.C.A Act. \& Div. Cient. 15(12): 373 - 379, 2012

\section{RESUMEN}

Se llevó a cabo una investigación para estimar un modelo de medición no destructiva de área foliar en plantas de arándano (Vaccinium corymbosum), en condiciones de trópico alto, mediante análisis secuencial de regresión, a partir de mediciones lineales de ancho de lámina foliar (Ah), longitud foliar (Lh) y el producto de la longitud por el ancho (LxA). Se utilizaron plantas de las variedades Sharp Blue y Biloxi para desarrollar los modelos en las localidades de Sibaté y de Firavitoba. El proceso de calibración fue desarrollo con las plantas sembradas en Sibaté, con 180 observaciones y, la validación de modelos, se efectuó en un huerto de Firavitoba. Se obtuvieron seis modelos, tres lineales y tres de forma polinómica de segundo orden, para cada material genético. Las formas seleccionadas fueron: $A F=0,638(L x A), R^{2} 0,94$ y $A F=0,0075(L x A)^{2}+0,3321(L x A)+2,2104, R^{2} 0,94$. Estos modelos fueron validados por nuevas muestras de hojas obtenidas de diferentes partes del dosel vegetal (bajo, medio y superior), solamente en plantas de la variedad Sharp Blue. Los modelos de la variedad Biloxi presentaron $\mathrm{R}^{2}$ muy bajos y alto CME. Los modelos, producidos en este estudio, se pueden utilizar de forma confiable para calcular el área individual de la hoja de distintos niveles foliares del dosel vegetal para la variedad de arándano Sharp Blue y, de esta forma, determinar el área foliar por planta sin necesidad de realizar muestreo destructivo. Esto no se puede aplicar para el cultivar Biloxi.

Palabras clave. Trópico alto, regresión, modelo lineal, frutales, Ericaceae.

\section{SUMMARY}

A research was carried out in order to estimate a leaf area model for blue berry (Vaccinium corymbosum) in high altitude tropical conditions, using linear measurements such as lamina length (L), width (W) and the length-width product (LxW) by stepwise regression analysis. Leaves from Sharp Blue and Biloxi cultivars were used to develop the model in the localities of Sibate and Firavitoba. The calibration step was developed with plants coming from Sibaté, with 180 observations and the models validation was effectuated at Firavitoba. Six models were obtained, three linear and three polynomial of second order, for each genetic material. The selection model forms were, $L A=0.638(L W), R^{2} 0.94$, and $\mathrm{LA}=0.0075(\mathrm{LW})^{2}+0.3321(\mathrm{LW})+2.2104, \mathrm{R}^{2}$ 0.94. These models were validated by measuring new leaf samples taken from different plant levels (low, medium and superior) of, only, the cultivar Sharp Blue. The models of Biloxi presented very low $\mathrm{R}^{2}$ and high CME. The models produced in this study can be used reliably for estimating the individual leaf area coming from different levels of the vegetal canopy of the blueberry cv Sharp Blue, and also, estimate the total plant leaf area, without destructive method. They are not recommendable for the Biloxi cultivar.

Key words: High tropic, linear regression, linear model, fruits, Ericaceae.

\section{INTRODUCCIÓN}

El arándano o mirtillo (Vaccinium corymbosum) es una planta originaria de Norte América, que forma parte del grupo de las frutas denominadas comercialmente, como berries (Van der Kloet \& Mcrae, 2009). Es un arbusto de follaje ca- 
duco de la familia de las Ericáceas, que posee numerosas especies, de las cuales, las más cultivadas son el tipo alto, Southern Highbush, (especie que primero se adaptó agronómicamente, con alrededor de 50 variedades) y ojo de conejo o Rabbiteye (Sonnentag et al. 2008).

La planta, se considera un frutal caducifolio, que requiere acumular determinadas cantidades de horas frío para salir del estado de reposo fisiológico obligado, debido a las condiciones invernales de los lugares de origen (Pavlovski, 2010; Mingeau et al. 2001). Esta condición limita el cultivo y la adaptación agronómica de la mayoría de variedades cultivados en latitudes cercanos al trópico; sin embargo, en la zona tropical alta, por encima de $2300 \mathrm{msnm}$, la temperatura mínima promedio del aire puede ser suficiente para que, variedades con bajo requerimiento de frío, puedan ser plantadas. La fruta de los arándanos es una baya esférica de color azul a negro, sabor agridulce, muy jugoso y aromático. Esta fruta tiene una importancia comercial significativa, con una demanda alta, producto de la divulgación de su utilización, como fuente de longevidad y como parte del tratamiento de enfermedades coronarias y degenerativas, debido a sus altos contenidos de antocianinas y polifenoles (Scalzo et al. 2008; Yang et al. 2009).

La demanda permanente de la fruta hizo que se establecieran nuevas zonas en la parte septentrional de América, especialmente, en Chile y en Argentina. En estos países, la producción de fruta está condicionada por la temperatura baja, lo cual, solamente permite una cosecha al año, normalmente, en noviembre y en enero (Lobos et al. 2009). El resto del año, la oferta de fruta es muy baja y la demanda crece permanentemente, hecho que provoca un desbalance comercial de gran impacto (Litwinczuk et al. 2005).

Actualmente, en Colombia se llevan a cabo algunos esfuerzos para adaptar materiales de arándano con bajos requerimientos de frío, inferiores a 350 Horas Frío, con posibilidades de producir fruta en épocas de contra estación con las zonas productoras del sur y del norte del continente americano (Paylis, 2004).

La adaptación de las plantas cultivadas obedece a una interacción genotipo por ambiente, donde la oferta ambiental determina, en gran medida, las posibilidades de uso y de explotación del recurso genético. Para realizar estudios de adaptación, se requieren estrategias metodológicas, que garanticen el logro de objetivos precisos y realizables. Uno de ellos es la caracterización del dosel de las plantas. El índice de área foliar, entendido como la razón entre el área de todas las hojas y el área del suelo ocupado por la planta, es uno de los indicadores más importantes en el crecimiento vegetal y en la producción de cosechas (Beerling \& Fray, 1990; Gutezeit, 2000). Para calcularlo, normalmente, se requieren mediciones destructivas o, de lo contrario, la utilización de equipos costosos que no siempre están disponibles en condiciones de campo (Bozhinova, 2006; Ajayi, 1990).

La determinación del área foliar por métodos indirectos es una herramienta de alta utilidad para evitar el muestreo destructivo, así como también sustituir el uso de equipos costosos y de alta sensibilidad. Los resultados de dicho método son muy acertados y de alta precisión, demostrándose su utilidad en varios cultivos, como pimentón (De Swart et al. 2004), kiwi (Mendoza de Gyves et al. 2007), haba (Peksen, 2007), cerezas (Cittadini \& Peri, 2006), calabacín (Rouphael et al. 2005), forestales (Cabezas-Gutiérrez et al. 2009), fresa (Demirsoy et al. 2005), banana (Zucoloto et al. 2008) y guayaba (Singh, 2007), entre otros. La gran mayoría de modelos obtenidos con el método son de tipo lineal, donde solamente se requieren mediciones elementales de largo de la lámina foliar, ancho promedio de la misma o una combinación de los dos atributos morfológicos (Kawamura \& Takeda, 2002).

El presente documento tiene como propósito mostrar una metodología para estimar el área foliar de dos materiales de Vaccinium corymbosum en condiciones de trópico alto, con el fin de contribuir con los estudios de caracterización ecofisiológica de la especie.

\section{MATERIALES Y MÉTODOS}

Se seleccionaron plantas de arándano (V. corymbosum) de dos años de edad, provenientes de la Empresa Genberries S.A., de Chile. Se escogieron dos variedades, Sharp Blue y Biloxi, que pertenecían a experimentos de adaptación de dichos materiales, en dos localidades de clima frío en Colombia: una, Sibaté, en Cundinamarca y la otra, Firavitoba, en Boyacá. El lote sembrado en Sibaté estaba localizado a 2780 msnm, con temperatura promedio de $12,5^{\circ} \mathrm{C}$, con variaciones térmicas entre 6 y $19^{\circ} \mathrm{C}$, respectivamente, para temperaturas mínima y máxima. El experimento de Firavitoba, se encontraba a $2480 \mathrm{msnm}$, con temperatura media de $14,5^{\circ} \mathrm{C}$, con variaciones térmicas de 7 y $22^{\circ} \mathrm{C}$. La precipitación de Sibaté es de $960 \mathrm{~mm}$ anuales y se presentan en promedio $1100 \mu \mathrm{mol} \cdot \mathrm{m}^{-2} \cdot \mathrm{s}^{-1}$ de densidad de flujo fotónico fotosintético (DFFT). En Firavitoba, se reportan en promedio $750 \mathrm{~mm}$ de precipitación media anual y $1540 \mu \mathrm{mol} \cdot \mathrm{m}^{-2} \cdot \mathrm{s}^{-1}$ de DFFT. Los datos meteorológicos, se midieron en cada localidad, mediante un termohidrómetro, marca HOBO 2134, para la temperatura del aire y una barra cuántica, marca LICOR 192, para la densidad de flujo de fotones. Los promedios de precipitación, se tomaron de los datos históricos del Instituto de Hidrología, Meteorología y Estudios Ambientales IDEAM (2011).

Las plantas estaban sembradas en camas levantadas a una distancia de $3 \mathrm{~m}$ entre sí y con una distancia de siembra de 
$1 \mathrm{~m}$ entre plantas, lo cual, arroja como resultado una población equivalente a 3333 plantas por hectárea. Todas las camas estaban provistas de un sistema de riego por goteo. Las plantas, durante el desarrollo del ensayo, fueron fertilizadas con una solución completa, que contenía $200 \mathrm{mg} \cdot \mathrm{L}^{-1}$ de $\mathrm{N}$, 30 de $\mathrm{P}, 150$ de $\mathrm{K}, 100$ de $\mathrm{Ca}, 15$ de S y 30 de $\mathrm{Mg}$, vía sistema de fertirrigación. Se aplicaron boro y hierro en forma foliar, con frecuencia mensual, en concentraciones de $0,1 \mathrm{~g} \cdot \mathrm{L}^{-1}$ de solubor y $1 \mathrm{~g} \cdot \mathrm{L}^{-1}$ de quelato de hierro, como fuentes de los micronutrientes. Las plantas, se mantuvieron libres de competencia por especies nocivas; se realizaron controles preventivos para enfermedades foliares, de acuerdo a los protocolos de la empresa hibridadora.

El trabajo, se dividió en dos partes: una, para establecer y calibrar los modelos y, la otra, para validarlos. En la localidad de Sibaté, se realizó la primera parte, mientras que la validación de los modelos, se efectuó con las plantas de la localidad de Firavitoba.

Calibración. La calibración y la construcción de los modelos, se llevó a cabo con la medición en el sitio experimental de 180 hojas por cada variedad, Sharp Blue y Biloxi, durante dos momentos del cultivo: uno, en estado de crecimiento foliar y, otra medición, en estado de floración. Se retiraron las hojas de diferentes tamaños y distintas posiciones dentro del dosel y con un analizador portátil, tipo CID 202 Portátil Leaf Área Meter, con $0,1 \mathrm{~mm}^{2}$ de resolución, se midió el área foliar; el largo y el ancho, se calcularon con una regla graduada en $\mathrm{mm}$. Con los datos de largo de la lámina foliar (Lh), ancho máximo de la lámina foliar (Ah) y el producto del largo por el ancho (LhxAh), se procedió a realizar los respectivos análisis de regresión lineal y polinomial, para las variables en referencia, respecto al área individual de la hoja, (Samuels \& Witmer, 2003). Los datos fueron procesados mediante el uso del programa EXCEL de Microsoft Office 2007®.

A partir de los resultados encontrados, se escogieron los modelos que presentaron los mayores coeficientes de determinación $\left(\mathrm{R}^{2}\right)$ y los menores cuadrados medios del error (CME), en concordancia con el procedimiento usado por Cittadini \& Peri (2006) y Mendoza de Gyves et al. (2007). La variedad Biloxi mostró bajos coeficientes de determinación, menores a 0,6, por tal razón, no se realizó la validación; mientas que Sharp Blue presentó $R^{2}$ más alto (mayor a 0,8) en varios de los modelos probados, aspecto que permitió realizar la validación en la otra localidad.

Validación. Una vez seleccionados los modelos de la previa calibración, se procedió a realizar la medición en la localidad número dos (Firavitoba). Allí, se seleccionaron 100 hojas de diferentes tamaños y de ramas que presentaban estados fenológicos entre desarrollo vegetativo y floración. Se separaron las hojas de las plantas y, en el sitio experimental, se ejecutó la medición con el mismo instrumento utilizado en la calibración. El área foliar individual obtenida y el área foliar estimada, a partir de las ecuaciones de cada modelo, fueron sometidas a un análisis de regresión lineal, para establecer la relación entre los datos obtenidos con cada modelo. La pendiente y el intercepto de cada modelo fueron probados mediante un análisis de varianza de una vía, para ver el nivel de significancia entre ellos, con base en la metodología empleada por Nautival et al. (1990) y (Peksen, 2007).

\section{RESULTADOS Y DISCUSIÓN}

Calibración de los modelos. De forma general, se encontró que el área foliar individual para la variedad Sharp Blue, varío entre $4,6 \mathrm{~cm}^{2}$ y $24,8 \mathrm{~cm}^{2}$, con promedio de $12,4 \mathrm{~cm}^{2}$, en Firavitoba y $11 \mathrm{~cm}^{2}$, en Sibaté. El largo de la lámina foliar varió entre 2,3 y $6,8 \mathrm{~cm}$, mientras el ancho lo hizo entre 1,4 y $5,2 \mathrm{~cm}$. La variedad Biloxi tuvo, en promedio, menor área foliar individual, $7,6 \mathrm{~cm}^{2}$ y presentó mayores variaciones entre largo y ancho de la lámina. De hecho, al realizar el análisis de datos, se halló que ninguno de los modelos aplicados en el presente trabajo presentó coeficientes confiables para replicar el modelo y realizar su validación en la variedad Biloxi.

En la tabla 1, se pueden observar las características de cada uno de los modelos establecidos para la estimación del área individual de las hojas del arándano, variedad Sharp Blue. Las ecuaciones presentadas, que corresponden a la variable área foliar individual, respecto al largo de la lámina foliar (L), ancho de la lámina foliar (A) y el producto AXL. Al realizar el ajuste estadístico, se observa que en todos los tres casos existe un coeficiente de determinación más alto, cuando se aplica el modelo de tipo polinómico, que al ser aplicado el modelo lineal.

Las ecuaciones demuestran que al utilizar un solo atributo, como base de la modelación, los coeficientes de determinación son bajos, menores a 0,8 , lo que ofrece poca confiabilidad para ser usado el modelo. También, se observa que al incrementar, ya sea el largo o el ancho de la lámina foliar, existe más bien una tendencia a estabilizarse el valor del área individual de la hoja. La forma entre ovoide y lanceolada de la hoja del arándano no facilita la obtención de una ecuación lineal para el ancho o el largo de la lámina foliar. Por tal razón, es requisito fundamental multiplicar ambos atributos para obtener un dato más confiable. Tanto el modelo 3 como el 6 , integran las dos variables y, en ambos casos, el coeficiente de determinación es superior a 0,9, una condición ideal a la hora de seleccionar los modelos adecuados para la estimación, como lo mencionan Bange et al. (2000), Bozhinova (2006), Gutiérrez \& Lavin (2000), en sus respectivos trabajos. Bajo estos argumentos, se seleccionaron los modelos 3 y 6 para realizar la validación en otra oferta ambiental. 
Tabla 1. Tipos de modelo utilizados para determinar el área foliar del arándano variedad Sharp Blue, en condiciones de trópico alto

\begin{tabular}{|c|l|c|c|c|}
\hline \multicolumn{5}{|c|}{ VARIEDAD SHARP BLUE } \\
\hline MODELO & \multicolumn{1}{|c|}{ TIPO } & ECUACIÓN & $\mathbf{R}^{2}$ & CME $\left(\mathbf{c m}^{2}\right)$ \\
\hline 1 & AF: $\mathrm{a}+\mathrm{bL} *$ & $\mathrm{y}=4,3622 \mathrm{x}-10,865$ & 0,7384 & 5,23 \\
\hline 2 & AF: $\mathrm{a}+\mathrm{bA}$ & $\mathrm{y}=5,0393 \mathrm{x}-6,6806$ & 0,7963 & 4,654 \\
\hline 3 & AF: $\mathrm{a}+\mathrm{b}(\mathrm{AL})$ & $\mathrm{y}=0,6385 \mathrm{x}-0,5164$ & 0,9351 & 1,278 \\
\hline 4 & AF: $\mathrm{a}+\mathrm{b}_{\mathrm{o}}(\mathrm{L})+\mathrm{b}_{1}(\mathrm{~L})^{2}$ & $\mathrm{y}=1,3412 \mathrm{x}^{2}-9,3924 \mathrm{x}+23,317$ & 0,8027 & 4,087 \\
\hline 5 & AF: $\mathrm{a}+\mathrm{b}_{\mathrm{o}}(\mathrm{A})+\mathrm{b}_{1}(\mathrm{~A})^{2}$ & $\mathrm{y}=1,2427 \mathrm{x}^{2}-3,8918 \mathrm{x}+8,5635$ & 0,823 & 3,676 \\
\hline 6 & AF: $\mathrm{a}+\mathrm{b}_{\mathrm{o}}(\mathrm{AL})+\mathrm{b}_{1}(\mathrm{AL})^{2}$ & $\mathrm{y}=0,0075 \mathrm{x}^{2}+0,3321 \mathrm{x}+2,2104$ & 0,9406 & 1,253 \\
\hline
\end{tabular}

* $\mathrm{L}=$ largo; $\mathrm{A}=$ ancho; $\mathrm{CME}=$ Cuadrado medio del error experimental.

Para el caso de la variedad Biloxi, ninguno de los modelos probados representa significancia, como tampoco exactitud para ser tenidos en cuenta en la determinación indirecta del área foliar (Tabla 2). Esta variedad presenta una alta variación en la forma de las hojas, algunas redondas, otras ovadas, otras lanceoladas y otras elípticas, lo cual, no permite hacer un ajuste matemático ideal para ser validado. Por tal razón, la variedad no se tuvo en cuenta para la validación de los modelos y, más bien, se propone medir el área foliar con métodos tradicionales hasta estimar un promedio lo suficientemente confiable para ser replicado en otras condiciones.

Validación: Una vez seleccionados los modelos, se calculó el área individual de las hojas, con base en los datos de longitud por ancho foliar, comparando este resultado con el dato obtenido por medición directa. El modelo lineal AF: $a+b(A L)$, presentado en la figura 1 , mostró un coeficiente de determinación de 0,93, considerado apropiado, respecto a lo encontrado en especies frutales por otros autores, como Serdar \& Demirsoy (2006), en castaño, Singh (2007), en guayabo y Cittadini \& Peri (2006) en cerezo. Para el modelo de tipo polinómico, el $\mathrm{R}^{2}$ fue de 0,94 , ligeramente superior, pero no significativamente, respecto al modelo lineal, figura 2 .

Si bien es cierto que en otras especies, como Kiwi (Mendoza de Gyves et al. 2007), calabacín (Rouphael et al. 2005), fresas (Demirsoy et al. 2005), algodón (Akram-Ghaderi \& Soltani, 2007) y brócoli (Gutezeit, 2000), los coeficientes de determinación son superiores, muy cercanos a uno; esas especies presentan alta homogeneidad en las muestras, pues, por lo general, las observaciones equivalen a materiales con alta homogeneidad en las formas de las hojas. Para el caso de arándano, las formas de la hoja varían entre plantas y entre estratos foliares, que puede ser la causa del presente resultado.

En términos generales, tanto el modelo lineal que involucra el producto del largo por el ancho de la hoja como el modelo polinómico de segundo orden, con los mismos componentes, pueden ser usados con alto nivel de confiabilidad para

Tabla 2. Tipos de modelo utilizados para determinar el área foliar del arándano variedad Biloxi.

\begin{tabular}{|c|l|c|c|c|}
\hline \multicolumn{5}{|c|}{ VARIEDAD BILOXI } \\
\hline MODELO & \multicolumn{1}{|c|}{ TIPO } & ECUACIÓN & $\mathbf{R}^{2}$ & CME(cm $\left.{ }^{2}\right)$ \\
\hline 1 & AF: $\mathrm{a}+\mathrm{bL} *$ & $\mathrm{y}=1,8619 \mathrm{x}-1,8072$ & 0,5306 & 3,589 \\
\hline 2 & AF: $\mathrm{a}+\mathrm{bA}$ & $\mathrm{y}=3,269 \mathrm{x}-2,1824$ & 0,5702 & 4,980 \\
\hline 3 & AF: $\mathrm{a}+\mathrm{b}(\mathrm{AL})$ & $\mathrm{y}=0,5434 \mathrm{x}+0,4238$ & 0,7424 & 3,345 \\
\hline 4 & AF: $\mathrm{a}+\mathrm{b}_{\mathrm{o}}(\mathrm{L})+\mathrm{b}_{1}(\mathrm{~L})^{2}$ & $\mathrm{y}=0,2694 \mathrm{x}^{2}-0,2155 \mathrm{x}+2,0979$ & 0,5373 & 5,587 \\
\hline 5 & AF: $\mathrm{a}+\mathrm{b}_{\mathrm{o}}(\mathrm{A})+\mathrm{b}_{1}(\mathrm{~A})^{2}$ & $\mathrm{y}=-4 \mathrm{E}-06 \mathrm{x}^{2}+3,269 \mathrm{x}-2,1824$ & 0,5702 & 3,480 \\
\hline 6 & AF: $\mathrm{a}+\mathrm{b}_{\circ}(\mathrm{AL})+\mathrm{b}_{1}(\mathrm{AL})^{2}$ & $\mathrm{y}=0,003 \mathrm{x}^{2}+0,4852 \mathrm{x}+0,6842$ & 0,7426 & 2,348 \\
\hline
\end{tabular}

$* \mathrm{~L}=$ largo; $\mathrm{A}=$ ancho. $\mathrm{CME}=$ cuadrado medio del error experimental 
estimar el área foliar individual en plantas de arándano de la variedad Sharp Blue, para evitar el muestreo destructivo en estudios fisiológicos y agronómicos en trópico alto. Para la variedad Biloxi, no se recomienda el uso de esta metodología de estimación, pero se sugiere realizar un nuevo trabajo que involucre ecuaciones diferentes a las probadas en este documento.
Agradecimiento: A la Universidad de Ciencias Aplicadas y Ambientales U.D.C.A, por la financiación. Conflicto de intereses: El manuscrito fue preparado y revisado con la participación de todos los autores, quienes declaramos que no existe ningún conflicto de intereses que ponga en riesgo la validez de los resultados presentados.

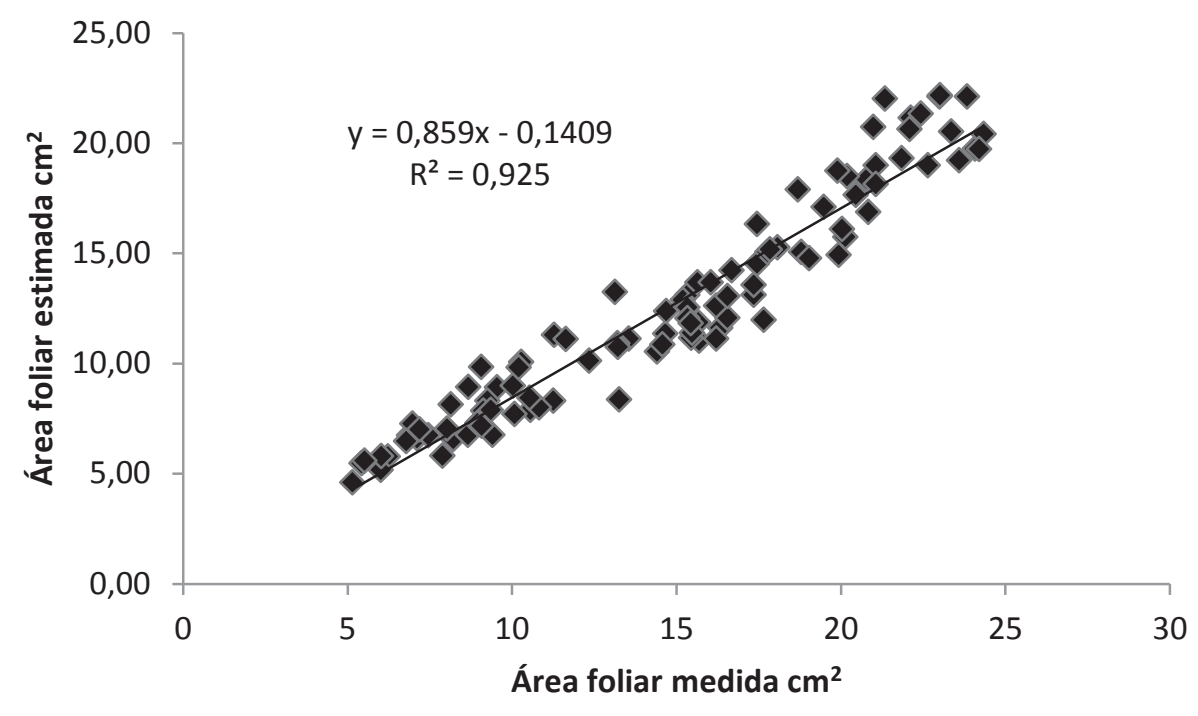

Figura 1. Relación entre el área foliar individual de hojas medida y el área foliar estimada en arándano variedad Sharp Blue, bajo condiciones de trópico alto. La estimación de área foliar, se realizó con el modelo lineal de tipo AF: a+b(AL).

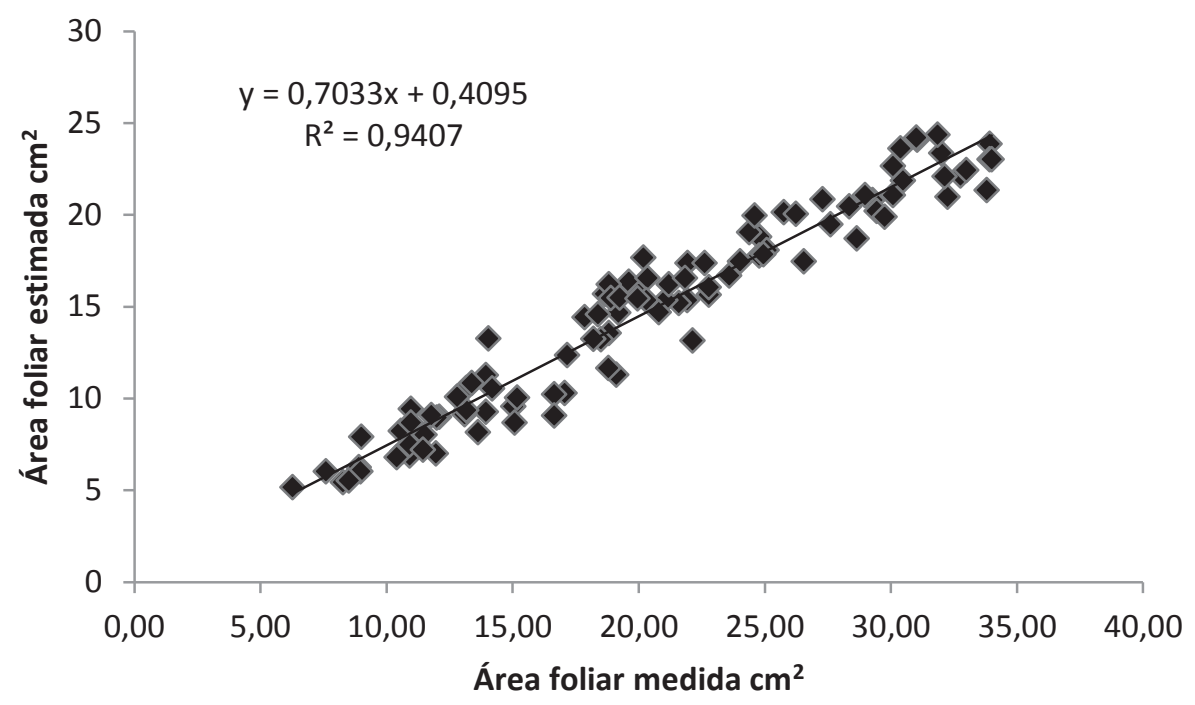

Figura 2. Relación entre el área foliar individual de hojas medida y el área foliar estimada en arándano variedad Sharp Blue, bajo condiciones de trópico alto. La estimación de área foliar, se realizó con el modelo lineal de tipo AF = a +bo(AL) +b1(AL)2. 


\section{BIBLIOGRAFÍA}

1. AJAYI, N.O. 1990. Rapid determination of leaf area in ovate vegetable leaves by linear measurements. J. Hort. Sc. 65(1):1-15.

2. AKRAM-GHADERI, M.; SOLTANI, F.A. 2007. Leaf area relationships to plant vegetative characteristics in cotton (Gossypium hirsutum L.) grown in a temperate subhumid environment. Int. J. Plant. Prod. $1(1): 63-71$

3. BANGE, M.P.; HAMMER, G.L; MILROY, S.P.; RICKERT. K. 2000. Improving estimates of individual leaf area of Sunflower. Agron. J. 92:761-765.

4. BEERLING, D.J.; FRAY, J.C. 1990. A comparison of the accurate variability and speed of five different methods for estimating leaf area. Ann. Bot. 65:483-488.

5. BOZHINOVA, R.P. 2006. Coefficients for determination of the leaf area in three burley. J. Central Eur. Agric. 7(1):7-12.

6. CABEZAS-GUTIÉRREZ, M.; PEÑA, J.P.; DUARTE, H.D.; COLORADO, J.F.; LORA, R. 2009. Un modelo para la estimación del área foliar en tres especies forestales de forma no destructiva. Rev. U.D.C.A, Act. \& Div. Cient. 12(1):121-130.

7. CITTADINI, E.D.; PERI, P.L. 2006. Estimation of leaf area in sweet cherry using a non-destructive method. RIA. 35(1):143-150.

8. DEMIRSOY, H.; DEMIRSOY, L.; ÖZTÜRK, A. 2005. Improved model for the non-destructive estimation of strawberry leaf area. Fruits. 60:69-73.

9. DE SWART, E.A.M.; GROENWOLD, R.; KANNE, H.J.; STAM, P.; MARCELLIS, L.F.M.; VOORRIPS, R.E. 2004. Non-destructive estimation of leaf area for different plant ages and accessions of Capsicum annum L. J. Hortic. Sci. Biotec. 79:764-770.

10. GUTEZEIT, B. 2000. Non-destructive measurement of fresh plant mass by the gamma-scanning technique applied to broccoli. J. Agric. Engng. Res. 75:251-255.

11. GUTIÉRREZ, T.A.; LAVÍN, A.A. 2000. Mediciones lineales en la hoja para la estimación no destructiva del área foliar en vides cv. Chardonnay. Agric. Téc. 60(1):9-17.
12. INSTITUTO DE HIDROLOGÍA, METEOROLOGÍA Y ESTUDIOS AMBIENTALES IDEAM. 2011. Información Hidrometeorológica de Colombia. Disponible desde Internet en: http://institucional.ideam.gov.co (con acceso 18/06/2011).

13. KAWAMURA, K.; TAKEDA, H. 2002. Light environment and crown architecture of two temperate Vaccinium species: inherent growth rules versus degree of plasticity in light response. Can. J. Bot. 80:1063-1077.

14. LITWINCZUK, W.; SZCZERBA, G.; WRONA, N. 2005. Field performance of highbush blueberries (Vaccinium corymbosum L.) cv. 'Herbert' propagated by cuttings and tissue culture. Sci. Hort. 106:162169.

15. LOBOS, G.A.; RETAMALES, J.B.; DEL POZO, A.; HANCOCK, J.F.; FLORE, J.A. 2009. Physiological response of Vaccinium corymbosum 'Elliott' to shading nets in Michigan. Acta Hort. 810:465-470.

16. MENDOZA DE GYVES, M.; ROUPHAEL, Y.; CRISTOFOTI, V.; MIRA, F.R. 2007. A non-destructive simple and accurate model for estimating the individual leaf area of kiwi (Actinidia deliciosa). Fruits. 62:171-175.

17. MINGEAU, M.; PERRIER, C.; AMEGLIO, T. 2001. Evidence of drought-sensitive periods from flowering to maturity on highbush blueberry. Sci. Hort. 89:2340.

18. NAUTIVAL, B.M.; SINGH, P.K.; SUKLA, R.N.; PRAKASH, S.; KUMAR, A. 1990. Correcting leaf area measurement by conventional methods: a new approach for apple (Malus domestica Borkh). J. Hort. Sc. 65(1):15-18

19. PAVLOVSKI, N. 2010. Influence of winter temperatures on the yielding ability of highbush blueberries (Vaccinium corymbosum L.) cultivars in Belarus. Internal J. Fruit Sci. 10:157-165.

20. PAYLIS, G.C. 2004. Blueberry fruit quality and yield as affected by fertilization. Acta Hort. 715:353-356.

21. PEKSEN, E. 2007. Non-destructive leaf area estimation model for faba bean (Vicia faba L.). Sci. Hort. 113:322-328.

22. ROUPHAEL, Y.; RIVERA, C.M.; CARDARELLI, M.; FANASCA, S.; COLLA, G. 2005. Leaf area estimation 
from linear measurements in zucchini plants of different ages. J. Hort. Sci. \& Biotechn. 81(2):238241.

23. SAMUELS, M.J.; WITMER, J.A. 2003. Statistics for the life sciences. $3^{\text {rd }}$ ed. Pearson Education, USA. 724p.

24. SCALZO, J.; CURRIE, A.; STEPHENS, J.; MCGHIE, T.; ALSPACH, P. 2008. The anthocyanin composition of different Vaccinium, Ribes and Rubus genotypes. BioFactors 34:13-21.

25. SERDAR, U.; DEMIRSOY, H. 2006. Non-destructive leaf area estimation in chestnut. Sci. Hort. 108:227230.

26. SINGH, A. 2007. Approximation of leaf area by using leaf dimensions in guava. I International Guava Symposium. Acta Hort. 735:321-324.

27. SONNENTAG, O.; TALBOT, J.; CHEN, J.M.; ROULET, N.T. 2008. Using direct and indirect measurements of leaf area index to characterize the shrub canopy in an ombrotrophic peatland. Agric. Forest. Meteorol. 144:200212.

28. VAN DER KLOET, S.P.; MCRAE, K.B. 2009. Stem longevity of Vaccinium corymbosum (Ericaceae) in Eastem North America. Am. Midi. Nat. 162:395-402.

29. YANG, W.Q.; HARPOLE, J.; FINN, C.E.; STRIK, B.C. 2009. Evaluating berry firmness and total soluble solids of newly released highbush blueberry cultivars. Acta Hort. 810(2):863-868.

30. ZUCOLOTO, M.; LIMA, J.S.S.; COELHO, R.I. 2008 Modelo matemático para estimativa da área foliar total de bananeira Prata-anã. Rev. Bras. Frut. 30:1152-1154.

Recibido: Junio 15 de 2012

Aceptado: Octubre 21 de 2012 